\title{
Evaluation of Degradation in Nanofilled Adhesive Resins Using Quantitative Light-Induced Fluorescence
}

\author{
Tae-Young Park, ${ }^{1}$ Sun-Jae Kim, ${ }^{1}$ Hee-Jung Kim, ${ }^{2}$ Byoung-Jin Lee, ${ }^{3}$ Byung-Hoon Kim, ${ }^{4}$ \\ Yeong-Mu Ko, ${ }^{4}$ and Jeong-Bum Min ${ }^{1}$ \\ ${ }^{1}$ Department of Conservative Dentistry, School of Dentistry, Chosun University, Gwangju 501-759, Republic of Korea \\ ${ }^{2}$ Department of Prosthodontic Dentistry, School of Dentistry, Chosun University, Gwangju 501-759, Republic of Korea \\ ${ }^{3}$ Department of Preventive Dentistry, School of Dentistry, Chosun University, Gwangju 501-759, Republic of Korea \\ ${ }^{4}$ Department of Dental Materials, School of Dentistry, Chosun University, Gwangju 501-759, Republic of Korea
}

Correspondence should be addressed to Jeong-Bum Min; minjb@chosun.ac.kr

Received 17 July 2014; Accepted 3 September 2014; Published 22 September 2014

Academic Editor: Seunghan Oh

Copyright (C) 2014 Tae-Young Park et al. This is an open access article distributed under the Creative Commons Attribution License, which permits unrestricted use, distribution, and reproduction in any medium, provided the original work is properly cited.

\begin{abstract}
The aim of this study was to evaluate degradation in commercial dental nanofilled adhesive resins using quantitative light-induced fluorescence (QLF). Three adhesives were selected: D/E resin (DR), Single Bond Plus (SB), and G-Bond (GB). The adhesives were mixed with porphyrin for the QLF analysis. Specimens were prepared by dispensing blended adhesives into a flexible mold and polymerizing. Then, the QLF analysis of the specimens was done and the porphyrin values (Simple Plaque Score and $\Delta R$ ) were measured. After thermocycling of the specimens $\left(5000\right.$ cycles, 5 to $55^{\circ} \mathrm{C}$ ) for the degradation, the specimens were assayed by QLF again. The porphyrin values were analyzed using paired $t$-test at a $95 \%$ confidence level. A significant reduction in SPS was observed in all groups after thermocycling. The $\Delta R$ significantly decreased after thermocycling except area $\Delta R 30$ of SB group. Overall, porphyrin values decreased after thermocycling which indicates that the degradation of the adhesive resins may be measured by the change of porphyrin value. The QLF method could be used to evaluate the degradation of adhesive resin.
\end{abstract}

\section{Introduction}

In 1982, the effectiveness of 4-methacryloxyethyltrimellitate anhydride (4-META) on the adhesion of an acrylic rod with etched dentine and enamel was first studied by Nakabayashi et al. [1]. This interlock was usually referred to as hybridization or the formation of a hybrid layer [1]. Since resin adhesion to dentin was first introduced in 1982, new adhesive resins have been developed to improve the bond strength. The structure of resin/dentin bond depends on the type of adhesive, and the various degradation of the bond may occur after time relapse [2]. Water sorption and solubility lead to the rapid and catastrophic degradation of resin/dentin bonds [2]. Therefore, a lot of methods were used for evaluating the degradation of these bonds. The use of Hoy's solubility parameters is typical method $[3,4]$, but such methods are not easy to use and they are time-consuming.
A quantitative light-induced fluorescence (QLF) device provides a clinical tool for the quantification of mineral loss and the detection of early dental caries from enamel in laboratory and clinical situations [5]. When illuminated with blue light ( $405 \mathrm{~nm}$ ), mature plaque produces red autofluorescence $[6,7]$. Such red autofluorescence has been observed using porphyrin. Therefore, a QLF device could not only detect porphyrin but also quantify the remaining porphyrin value. Porphyrin is a group of naturally occurring organic compounds. One of the best known porphyrins is heme, the pigment in red blood cells; heme is a cofactor of the protein hemoglobin [8]. As a photosensitizer, porphyrin has been used for localization and photodynamic therapy of neoplastic disease [9].

Water sorption and solubility are useful methods to evaluate the degradation of the dental resin. The loss of the resin by degradation was measured $[3,4]$. If the dental resin 
TABLE 1: Composition of the adhesive systems used in the study.

\begin{tabular}{ll}
\hline Adhesive (manufacturer) & Components (lot no.) \\
\hline $\begin{array}{l}\text { D/E resin } \\
\text { (BISCO, Schaumburg, IL, USA) }\end{array}$ & Bis-GMA, HEMA, and urethane dimethacrylates (1400001272) \\
\hline $\begin{array}{l}\text { Adper Single Bond Plus } \\
\text { (3M/ESPE, St. Paul, MN, USA) }\end{array}$ & $\begin{array}{l}\text { Bis-GMA, HEMA, dimethacrylates, polyalkenoic acid copolymer, initiators, water, } \\
\text { ethanol, and silica filler (N538311) }\end{array}$ \\
\hline $\begin{array}{l}\text { G-Bond } \\
\text { (GC, Tokyo, Japan) }\end{array}$ & $\begin{array}{l}\text { 4-MET, phosphoric ester monomer, UDMA, TEGDMA, acetone, water, stabilizer, silica } \\
\text { filler, water, and photoinitiator (1307091) }\end{array}$ \\
\hline
\end{tabular}

4-MET: 4-methacryloxyethyltrimellitate; UDMA: urethane dimethacrylate; TEGDMA: triethyleneglycol dimethacrylate; bis-GMA: bisphenol A diglycidyl ether dimethacrylate; and HEMA: 2-hydroxyethyl methacrylate.

contains porphyrin, the loss of porphyrin by degradation may be measured using QLF. However porphyrin has not yet been used for the evaluation of a restoration's durability and degradation.

The aim of this study was to evaluate the degradation of commercial dental nanofilled adhesive resins after thermocycling using QLF. The null hypothesis was that there would be no difference in the porphyrin values between prethermocycling and postthermocycling of the adhesive resin.

\section{Materials and Methods}

2.1. Specimen Preparation. Three adhesives were selected: $\mathrm{D} / \mathrm{E}$ resin (DR), Single Bond Plus (SB), and G-Bond (GB). $\mathrm{D} / \mathrm{E}$ resin, classified as a three-step etch and rinse adhesive, has no nanofillers. Single Bond Plus contains nanofillers and is classified as a two-step etch and rinse adhesive. GBond is a one-step self-etch adhesive containing nanofillers. The compositions and respective manufacturers of the three adhesives are shown in Table 1. Porphyrin I (Sigma, St. Louis, MO, USA) was mixed with the adhesives. GB and SB were vibrated with $1 \mathrm{mg}$ of porphyrin I and DR was also vibrated with $0.5 \mathrm{mg}$ of porphyrin I. Twenty resin disks $(n=20)$ for each group were fabricated in a mold ( $5 \mathrm{~mm}$ diameter, $0.8 \mathrm{~mm}$ thick). The liquid adhesive was directly dispensed to fully fill the silicon mold. The surfaces of the solvated adhesives, GB and SB, were gently blown with an oil/water-free compressed air for $90 \mathrm{~s}$ to facilitate solvent evaporation. A glass cover slide was placed on the top of the adhesives to exclude atmospheric oxygen and displace excess solution. Each specimen was immediately cured with a quartz tungsten halogen (QTH) lamp (Spectrum 800, Dentsply Caulk, Milford, DE, USA) for $40 \mathrm{~s}$. This lamp provided an irradiance of $400 \mathrm{~mW} / \mathrm{cm}^{2}$. After removing each specimen from the mold, photoactivation was repeated on its opposite surface for $40 \mathrm{~s}$. As control, pure resin disks without porphyrin were fabricated.

2.2. QLF Analysis and Thermocycling. The QLF device used was QLF-D (QLF-D Biluminator, Inspektor Research systems BV, Amsterdam, The Netherlands). The QLF-D Biluminator is a new type of QLF devices (Figure 1). Fluorescence images were captured with a digital full-sensor single-lens reflex (SLR) camera (model 550D, Canon, Tokyo, Japan) at the following settings: shutter speed of $1 / 45 \mathrm{~s}$, aperture value of 3.2, and ISO speed of 1600. Proprietary software (C3 v1.18, Inspektor Research Systems BV) was used to store all digital images on a PC automatically. All fluorescence images were analyzed using a computer program (QA2 v1.18, Inspektor Research Systems BV) by measuring the plaque patch.

A region of interest was defined by manually outlining an area on the surface using an interface within the capture software. Since the green fluorescence of teeth is of relatively uniform color, relatively plaque- (porphyrin-) free baseline images were used to generate a function $f$ describing this color, which expressed the value of the red channel $R$ as a quadratic function of the value of the green channel $G$. Red light passed through the disclosed plaque with very little absorption, so applying this function to the measured values of the green channel resulted in a good approximation of the red channel on clean areas of the tooth and an underestimate elsewhere. Pixels were classified as either containing plaque or clean by applying a threshold value $T$, such that any pixel where $R \div f(G)-1 \geq T$ is treated as plaque.

The final plaque (porphyrin) percentage coverage $(\Delta R)$ is the percentage of pixels within the defined area of the tooth surface classified as plaque. $\Delta R$ is the percentage of the increase of the ration of red and the green component with respect to the ratio of sound tissue. Different thresholds were applied on areas $\Delta R 30, \Delta R 70$, and $\Delta R 120$. The higher the number was, the higher the threshold value was. Thereby, the porphyrin value was correlated with the increasing intensity of red fluorescence.

The Simple Plaque Score (SPS) was automatically calculated from the fluorescence image. A 6-point (0-5) scoring system was defined as a value from 0 (no mature plaque) to 5 (high amount of mature plaque). Thermocycling was performed for 5000 cycles in deionized water from 5 to $55^{\circ} \mathrm{C}$ with a dwelling time of $30 \mathrm{~s}$ in each bath and a transfer time of $3 \mathrm{~s}$. After thermocycling, each specimen was assayed by QLF$\mathrm{D}$ in the same way.

2.3. Statistical Analysis. All data were analyzed by one-way ANOVA. The differences between pre- and postthermocycling were analyzed by paired $t$-test. All statistical procedures were performed using the SPSS 12.0 for Windows (IBM Corp., Armonk, NY, USA) and the level of significance was set at $P=0.05$. 


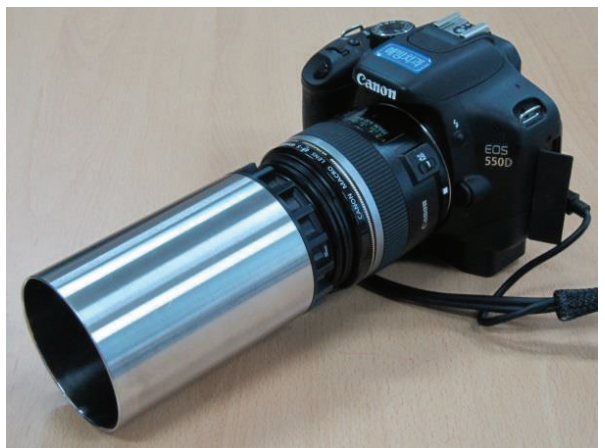

(a)

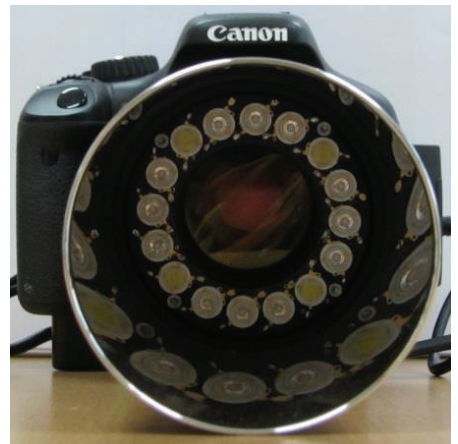

(b)

FIgURE 1: The QLF-D Biluminator used in this study. This device (a) is based on a full-sensor SLR camera (Canon 550D) equipped with an illumination tube with white and blue light-emitting diodes positioned in a ring around the lens opening (b).

TABLE 2: Simple Plaque Score (mean \pm SD).

\begin{tabular}{lccc}
\hline & DR & SB & GB \\
\hline Prethermocycling & $4.2 \pm 0.95^{\mathrm{a}}$ & $4.0 \pm 0^{\mathrm{a}}$ & $5.0 \pm 0.22^{\mathrm{a}}$ \\
Postthermocycling & $1.5 \pm 1.43^{\mathrm{b}}$ & $1.9 \pm 1.62^{\mathrm{b}}$ & $0.7 \pm 0.86^{\mathrm{b}}$ \\
\hline
\end{tabular}

Different lowercase letters indicate a significant difference between pre- and postthermocycling $(P<0.05)$.

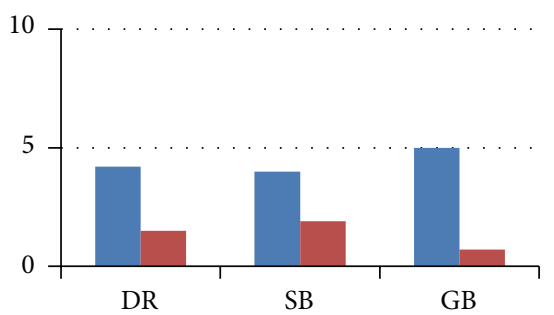

- Prethermocycling

- Postthermocycling

Figure 2: Graph presenting the changes in Simple Plaque Score. All groups showed significant reduction of SPS after thermocycling $(P<$ $0.05)$.

\section{Results and Discussion}

The QLF-D results are presented in Table 2 and Figure 3. All groups showed significant reduction of SPS after thermocycling (Table 2 and Figure 2).

The $\Delta R$ significantly decreased after thermocycling except area $\Delta R 30$ of SB (Table 3 and Figure 3). The porphyrin of resin disks in all groups almost disappeared.

The fluorescence images of the pre- and postthermocycled resin disks are given in Figure 4. All specimens had red autofluorescence before thermocycling. However, red autofluorescence decreased after thermocycling in all specimens. Red autofluorescence especially drastically decreased in the GB resin disk, in which SPS was 0 (Figure 4). GB resin disk was decolorized after thermocycling (Figure 5).
TABLE 3: $\Delta R$ (mean \pm SD, unit: \%).

\begin{tabular}{lccc}
\hline Area & DR & SB & GB \\
\hline$\Delta R 30$ & & & \\
Prethermocycling & $4.3 \pm 2.09^{\mathrm{a}}$ & $2.2 \pm 0.1^{\mathrm{a}}$ & $20 \pm 11.75^{\mathrm{a}}$ \\
Postthermocycling & $2.23 \pm 2.10^{\mathrm{b}}$ & $1.78 \pm 1.36^{\mathrm{a}}$ & $1.06 \pm 1.6^{\mathrm{b}}$ \\
$\Delta R 70$ & & & \\
Prethermocycling & $1.8 \pm 1.46^{\mathrm{a}}$ & $1.9 \pm 0.07^{\mathrm{a}}$ & $8.5 \pm 8.84^{\mathrm{a}}$ \\
Postthermocycling & $0.05 \pm 0.12^{\mathrm{b}}$ & $0.51 \pm 0.80^{\mathrm{b}}$ & $0.05 \pm 0.22^{\mathrm{b}}$ \\
$\Delta R 120$ & & & \\
Prethermocycling & $0.9 \pm 0.98^{\mathrm{a}}$ & $1.8 \pm 0.06^{\mathrm{a}}$ & $3.2 \pm 5.15^{\mathrm{a}}$ \\
Postthermocycling & $0 \pm 0^{\mathrm{b}}$ & $0.40 \pm 0.71^{\mathrm{b}}$ & $0 \pm 0^{\mathrm{b}}$ \\
\hline
\end{tabular}

Different lowercase letters indicate a significant difference between pre- and postthermocycling in each area $(P<0.05)$.

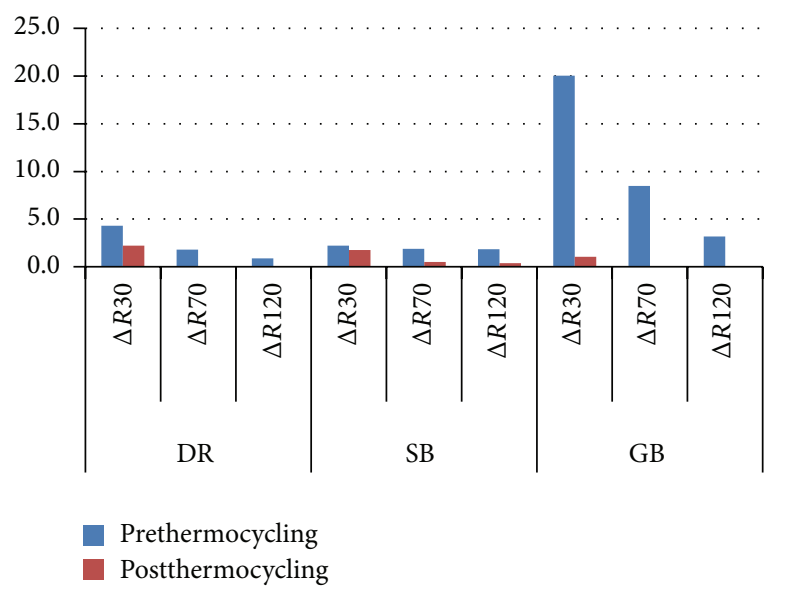

FIgURE 3: Graph presenting the changes in $\Delta R$. All groups showed significant reduction of $\Delta R$ after thermocycling except area $\Delta R 30$ of SB $(P<0.05)$.

If this degradation occurs in the resin/dentin bond interface, it could weaken tooth adhesion [10]. Therefore, there have been many attempts to simulate bond degradation in vitro [11]. Many results demonstrated that a critical factor affecting durability in vivo and in vitro was hydrolysis of 


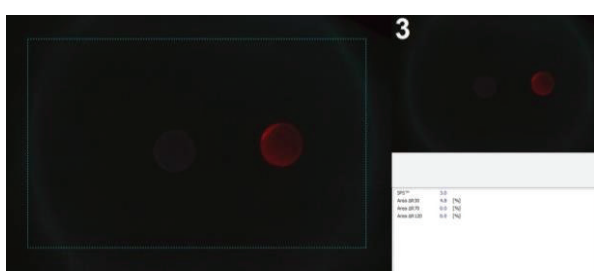

(a) Prethermocycling

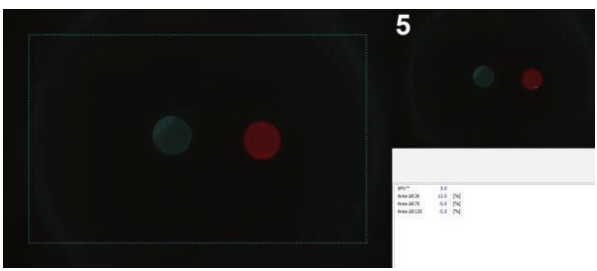

(c) Prethermocycling

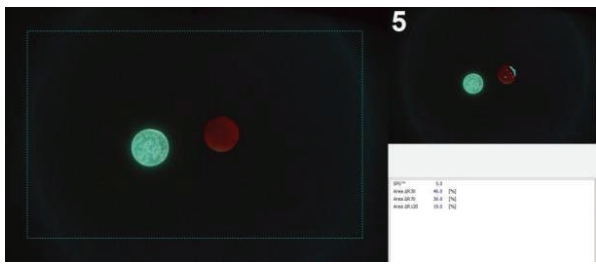

(e) Prethermocycling



(b) Postthermocycling

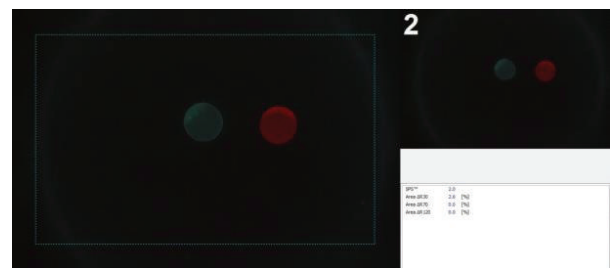

(d) Postthermocycling

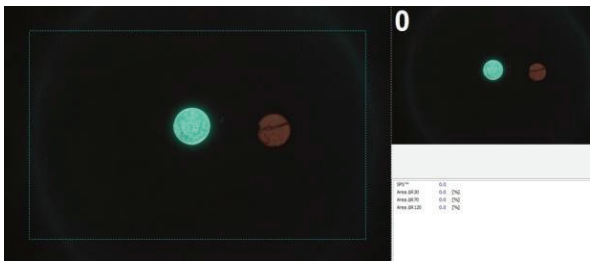

(f) Postthermocycling

FIGURE 4: QLF-D fluorescence images of (a, c, and e) pre- and (b, d, and f) postthermocycled resin disks. (a), (b) DR; (c), (d) SB; and (e), (f) GB.

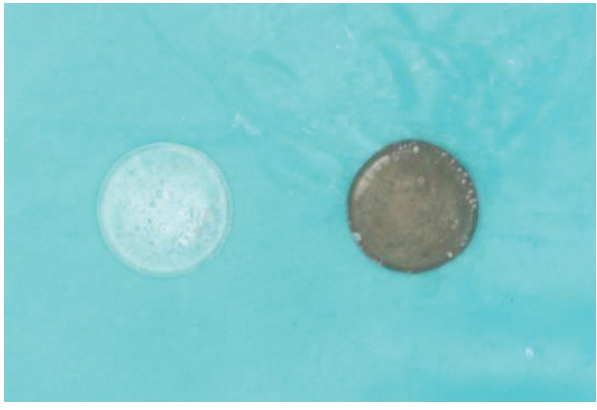

(a) Prethermocycling

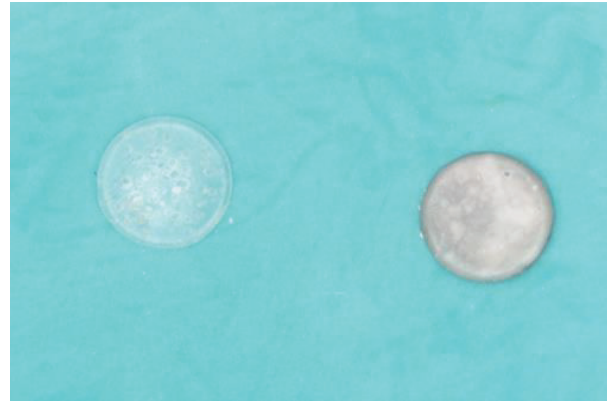

(b) Postthermocycling

FIGURE 5: Photographs of resin disks ( $\times 8$ ). (a) Resin disks before thermocycling: left disk is sound resin and right is GB resin. (b) Resin disks after thermocycling: left disk is sound resin and right disk is GB resin.

the resin/dentin interface such as collagen hydrolysis, resin elusion by hydrolysis, and the increasing amount of nanoleakage after aging [2]. Currently, the most validated method for the assessment of this degradation process in vitro is the storage of microspecimens in water [12]. Previous water storage studies measured their solubility and interfacial bond strength $[13,14]$. We attempt to suggest a valid and easier method. Our study tried to evaluate the actual degradation of the adhesive resin using porphyrin and QLF. The degradation of adhesive resin means the destruction of the polymers in cured adhesive. During this degradation process, porphyrin may be released from weakened polymers. This means that the degradation of the adhesive resins may be measured by the change of porphyrin value.

SB and GB have relatively highly hydrophilicity because of their compositions, a significant amount of solvent and hydrophilic monomers. DR is relatively hydrophobic. A previous study demonstrated that the most hydrophilic resin showed high water sorption, water solubility, and water diffusion coefficient and that the extent and rate of water sorption increased with increasing hydrophilicity of the resin blends [13]. However, all adhesive resins showed significant porphyrin loss after thermocycling (Tables 2 and 3). Their water solubility might contribute to the hydrolysis of adhesive 
resin after thermocycling. The hydrolysis of adhesive resins might cause loss of porphyrin, causing degradation of the adhesive resin. Another study demonstrated that the onestep self-etch adhesive and self-etch primer/adhesive mixtures presented the highest water sorption and solubility values [15]. Before thermocycling, the porphyrin value of the three adhesive resins was varied. GB contains TEGDMA, producing a highly crosslinked polymer network and water in order to ionize acidic monomers such as 4-MET and a phosphoric ester monomer. SB and DR contain Bis-GMA resin as cross-linkers. Bis-GMA includes both hydrophilic and hydrophobic components. The core diphenylisopropane presents significant hydrophobicity on Bis-GMA, whereas the two hydroxyl groups are the major source of hydrophilicity and they can bind via hydrogen bonding, resulting in absorbed water [16]. Because of the poor water solubility of Bis-GMA, manufacturers usually add acetone or HEMA to facilitate Bis-GMA solubility. Use of a HEMA-based primer prevents collagen collapse and rewets dry dentin [17]. Also, even in relatively low concentrations (5\% or $15 \%$ ), the addition of ethanol into the experimental methacrylatebased dental adhesives tested increased the ability of these materials to absorb and transport water [18]. The different compositions of three adhesive resins may be the cause of the variations in the loss of porphyrin value.

Several studies had shown that water absorption is dependent not only on the presence of the residual solvent but also on the degree of hydrophilicity of the materials [1416]. Another study showed that when the concentration of hydrophilic comonomers was high, the colligative properties of the entire mixture were changed, lowering the vapor pressure of the volatile components, such as nonpolymerizable solvents (i.e., acetone, ethanol, and water) [17]. The dimensions of the disks were selected to provide uniform homogenous samples to allow easy manipulation and avoid the risk of fracture during the experiment. However, these thick resin disks, especially those of SB and GB, might prevent complete solvent removal. Therefore, the presence of residual solvent in an adhesive resin during polymerization would enhance water sorption.

Nanofillers are added to enhance physical properties of adhesives resin. SB and GB contain nanofillers, but these could not prevent the loss of porphyrin values of SB and GB.

Most in vitro durability studies analyze one of the in vivo degradation factors involved in order to disclose its effect on the general degradation process, in contrast to the clinical situation in which all these factors operate simultaneously [12]. In addition, our results might be exaggerated because the resin disks were directly exposed to a hydrothermal state. Another problem was that thermocycling process might not reflect degradation within the mouth. Therefore, this limitation should be considered in future studies.

\section{Conclusions}

All groups in this study showed a significant decrease of porphyrin value. Therefore, the null hypothesis was rejected. Within the limits of this study, the QLF method may be used to evaluate the degradation of adhesive resin as a new method. This method may provide more information about the fundamental mechanisms involved in resin/dentin interface degradation.

\section{Conflict of Interests}

The authors declare that there is no conflict of interests regarding the publication of this paper.

\section{Acknowledgment}

This research was supported by the National Research Foundation of Korea (NRF) funded by the Ministry of Science, ICT and Future Planning (no. R13-2008-010-00000-0).

\section{References}

[1] N. Nakabayashi, K. Kojima, and E. Masuhara, "The promotion of adhesion by the infiltration of monomers into tooth substrates," Journal of Biomedical Materials Research, vol. 16, no. 3, pp. 265-273, 1982.

[2] M. Hashimoto, "A review-micromorphological evidence of degradation in resin-dentin bonds and potential preventional solutions," Journal of Biomedical Materials Research-Part B Applied Biomaterials, vol. 92, no. 1, pp. 268-280, 2010.

[3] R. G. Miller, C. Q. Bowles, C. C. Chappelow, and J. D. Eick, "Application of solubility parameter theory to dentinbonding systems and adhesive strength correlations," Journal of Biomedical Materials Research, vol. 41, no. 2, pp. 237-243, 1998.

[4] Y. Nishitani, M. Yoshiyama, K. Hosaka et al., "Use of Hoy's solubility parameters to predict water sorption/solubility of experimental primers and adhesives," European Journal of Oral Sciences, vol. 115, no. 1, pp. 81-86, 2007.

[5] I. A. Pretty, W. M. Edgar, and S. M. Higham, "The effect of ambient light on QLF analyses," Journal of Oral Rehabilitation, vol. 29, no. 4, pp. 369-373, 2002.

[6] L. Coulthwaite, I. A. Pretty, P. W. Smith, S. M. Higham, and J. Verran, "The microbiological origin of fluorescence observed in plaque on dentures during QLF analysis," Caries Research, vol. 40, no. 2, pp. 112-116, 2006.

[7] R. Z. Thomas, H. C. van der Mei, M. H. van der Veen, J. J. de Soet, and M. C. D. N. J. M. Huysmans, "Bacterial composition and red fluorescence of plaque in relation to primary and secondary caries next to composite: an in situ study," Oral Microbiology and Immunology, vol. 23, no. 1, pp. 7-13, 2008.

[8] K. M. Kadish, K. M. Smith, and R. Guilard, The Porphyrin Handbook, Elsevier Science, 2003.

[9] D. Kessel, Methods in Porphyrin Photosensitization, Plenum Publishing, 1985.

[10] M. R. O. Carrilho, R. M. Carvalho, F. R. Tay, and D. H. Pashley, "Effects of storage media on mechanical properties of adhesive systems," American Journal of Dentistry, vol. 17, no. 2, pp. 104108, 2004.

[11] F. L. B. Amaral, V. Colucci, R. G. Palma-Dibb, and S. A. M. Corona, "Assessment of in vitro methods used to promote adhesive interface degradation: a critical review," Journal of Esthetic and Restorative Dentistry, vol. 19, no. 6, pp. 340-353, 2007.

[12] J. de Munck, K. van Landuyt, M. Peumans et al., "A critical review of the durability of adhesion to tooth tissue: methods 
and results," Journal of Dental Research, vol. 84, no. 2, pp. 118132, 2005.

[13] J. Malacarne, R. M. Carvalho, M. F. de Goes et al., "Water sorption/solubility of dental adhesive resins," Dental Materials, vol. 22, no. 10, pp. 973-980, 2006.

[14] J. de Munck, B. van Meerbeek, Y. Yoshida et al., "Four-year water degradation of total-etch adhesives bonded to dentin," The Journal of Dental Research, vol. 82, no. 2, pp. 136-140, 2003.

[15] A. F. Reis, M. Giannini, and P. N. R. Pereira, "Influence of waterstorage time on the sorption and solubility behavior of current adhesives and primer/adhesive mixtures," Operative Dentistry, vol. 32, no. 1, pp. 53-59, 2007.

[16] D. Skrtic and J. M. Antonucci, "Effect of bifunctional comonomers on mechanical strength and water sorption of amorphous calcium phosphate- and silanized glass-filled Bis-GMAbased composites," Biomaterials, vol. 24, no. 17, pp. 2881-2888, 2003.

[17] L. Hitmi, D. Bouter, and M. Degrange, "Influence of drying and HEMA treatment on dentin wettability," Dental Materials, vol. 18, no. 7, pp. 503-511, 2002.

[18] J. Malacarne-Zanon, D. H. Pashley, K. A. Agee et al., "Effects of ethanol addition on the water sorption/solubility and percent conversion of comonomers in model dental adhesives," Dental Materials, vol. 25, no. 10, pp. 1275-1284, 2009. 

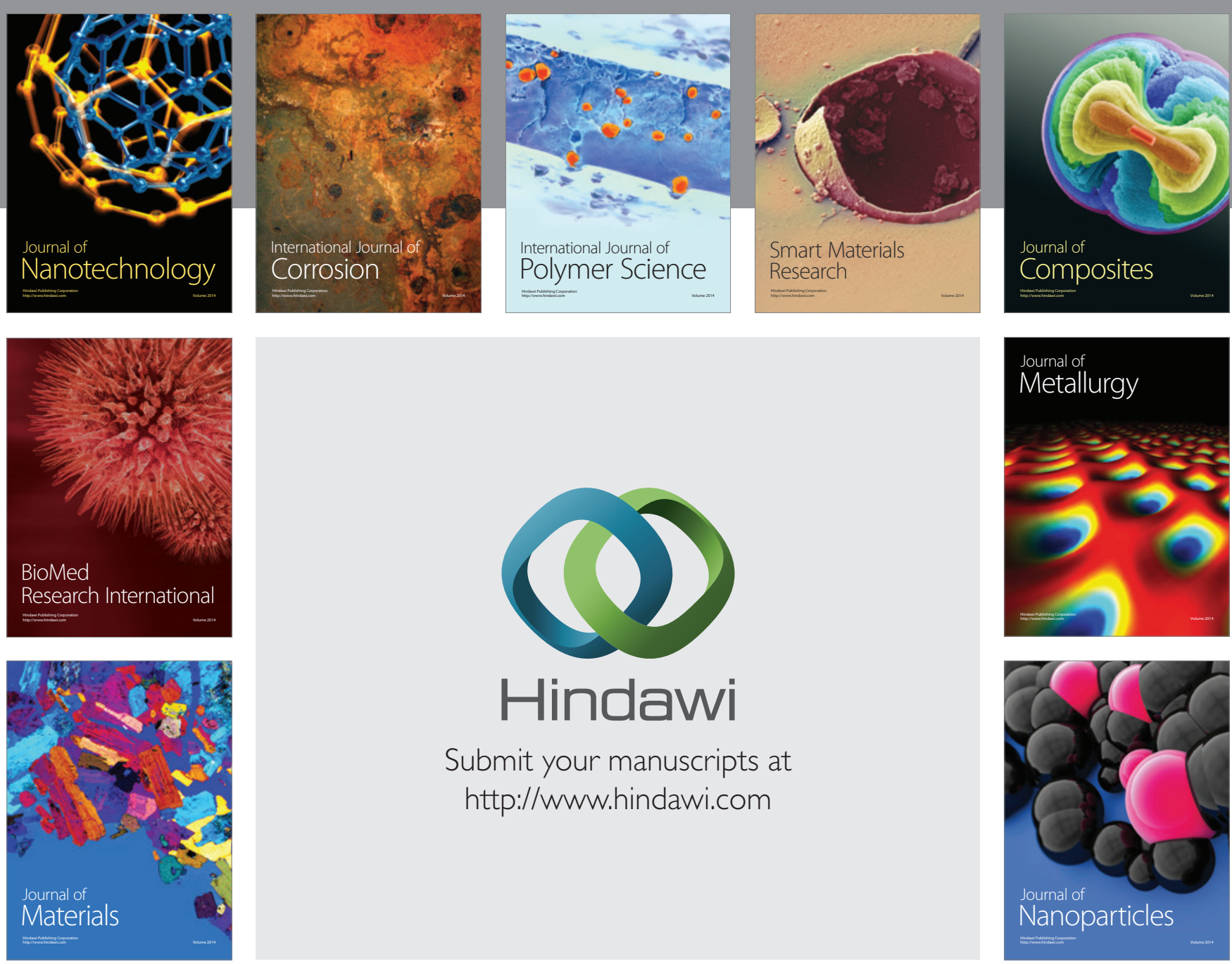

Submit your manuscripts at http://www.hindawi.com
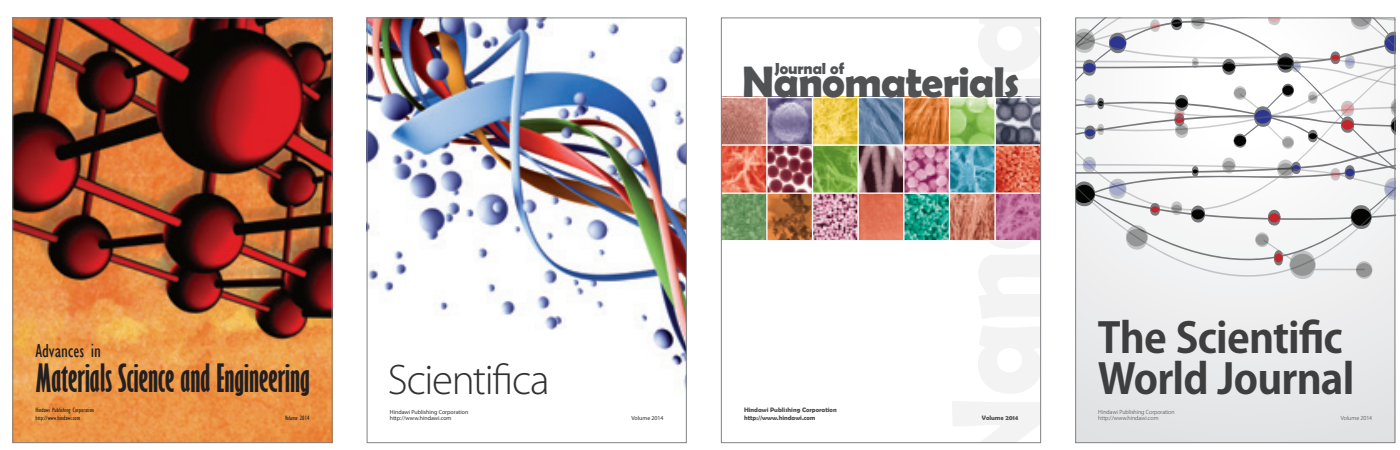

\section{The Scientific World Journal}
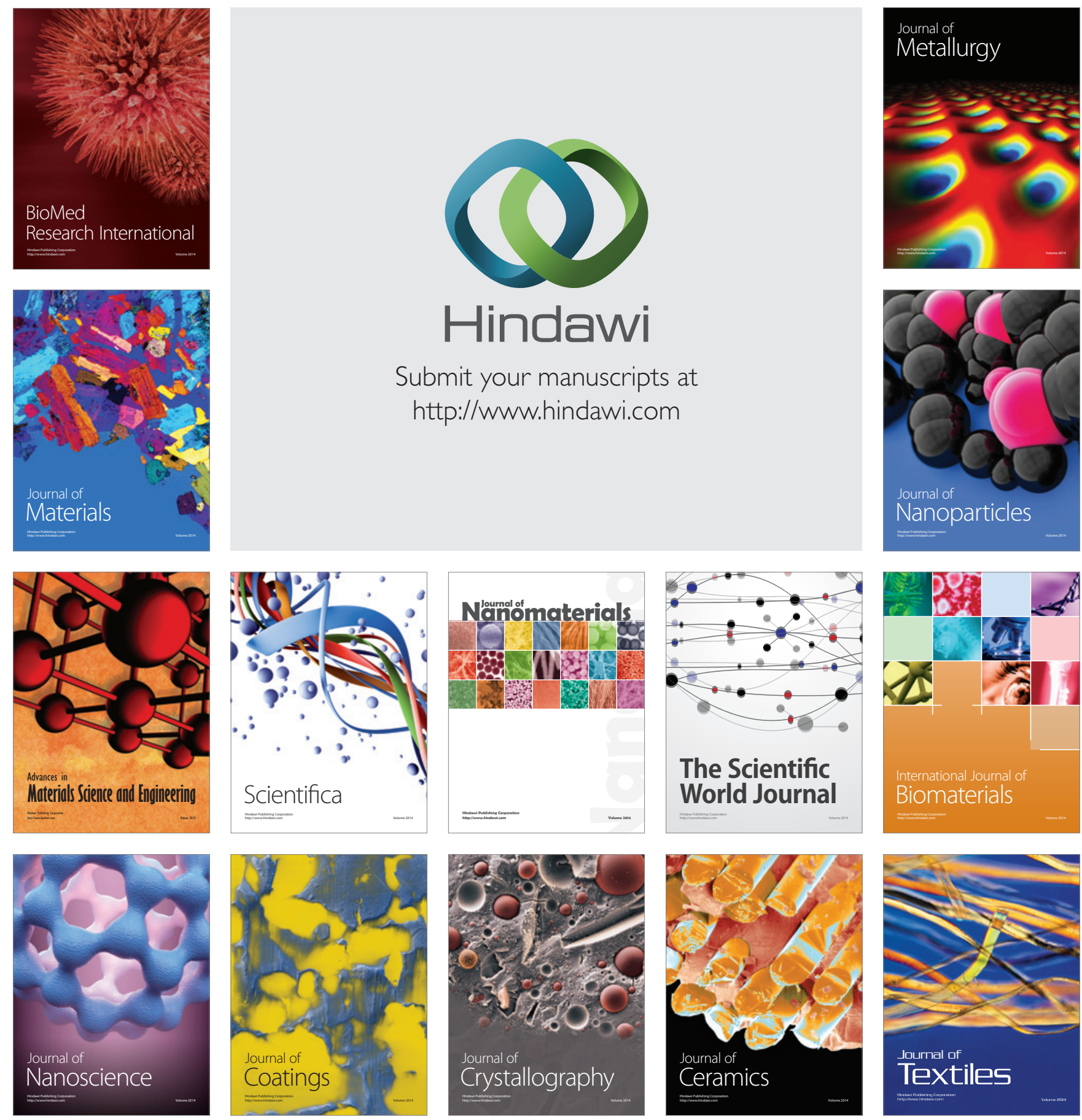